\title{
MRI T2 signal intensity and tumor response in patients with GH-secreting pituitary macroadenoma: PRIMARYS post hoc analysis
}

\author{
Fabrice Bonneville', Louis-David Rivière', Stephan Petersenn², John S Bevan ${ }^{3}$, Aude Houchard ${ }^{4}$, Caroline Sert ${ }^{4}$ \\ and Philippe J Caron ${ }^{5}$ on behalf of the PRIMARYS Study Group ${ }^{t}$
}

\begin{abstract}
${ }^{1}$ Department of Neuroradiology, CHU Purpan, Toulouse, France, ${ }^{2}$ ENDOC Center for Endocrine Tumors, Hamburg, Germany, ${ }^{3} \mathrm{JJR}$ Macleod Centre for Diabetes, Endocrinology \& Metabolism (Mac-DEM), Aberdeen Royal Infirmary, Aberdeen, UK, ${ }^{4}$ |psen, Boulogne Billancourt, France, and ${ }^{5}$ Department of Endocrinology \& Metabolic Diseases, Centre Hospitalier Universitaire Larrey, Toulouse, France

${ }^{\dagger}$ (Details of the PRIMARYS Study Group is presented in the Acknowledgement section)

Correspondence should be addressed to P J Caron

Email

caron.p@chu-toulouse.fr
\end{abstract}

\begin{abstract}
Objective: Pituitary adenoma MRI T2 signal intensity associates with tumor characteristics including responsiveness to somatostatin analogs (SSAs). These analyses determined whether baseline $\mathrm{T} 2$ signal intensity predicts response to primary medical treatment with long-acting SSA.

Design: Post hoc analyses of the prospective multicenter, open-label, single-arm PRIMARYS study in which patients with treatment-naïve GH-secreting pituitary macroadenomas received fixed-dose lanreotide autogel $(120 \mathrm{mg})$ every 4 weeks for 48 weeks.

Methods: Associations were investigated between adenoma T2-signal hypo/iso/hyperintensity and treatment responses at week 48/last visit: hormonal control (GH $\leq 2.5 \mu \mathrm{g} / \mathrm{L}$ and IGF-1 normalization); tumor response (tumor volume reduction (TVR) $\geq 20 \%$ ); separate GH/IGF-1 control and change from baseline in GH/IGF-1 and tumor volume. Results: Adenomas were hypointense at baseline in 50/85 (59\%) patients using visual assessment. Of these, 40\% achieved hormonal control and $76 \%$ achieved a tumor response. Significant univariate associations arose for hypo-vs isointensity with tumor response and achievement of GH $\leq 2.5 \mu \mathrm{g} / \mathrm{L}$, but not IGF-1 normalization or overall hormonal control. In multivariate analysis, tumor response was six times more likely for hypo- vs iso-intense tumors (=6.15; 95\% Cl: 1.36-27.88). In univariate change-from-baseline analyses, hypo-vs isointensity was associated with greater TVR and IGF-1 reduction but not change in GH. In multivariate analysis, IGF-1 decreased by an estimated additional $65 \mu \mathrm{g} / \mathrm{L}$ $(P=0.0026))$ for hypo- vs isointense.

Conclusions: Patients with hypointense vs isointense GH-secreting macroadenomas had greater reductions in IGF-1 following primary treatment with lanreotide autogel and were more likely to achieve tumor response. Assessment of T2 signal intensity at baseline may help to predict long-term responses to primary treatment with SSAs.
\end{abstract}

\section{Introduction}

After biochemical diagnosis of acromegaly, magnetic resonance imaging (MRI) of the underlying benign pituitary adenoma is required, according to clinical guidance from the Endocrine Society (1). Surgery, the first-line treatment for most patients, can be curative but results in normalization of insulin-like growth https://eje.bioscientifica.com https://doi.org/10.1530/EJE-18-0254 Printed in Great Britain

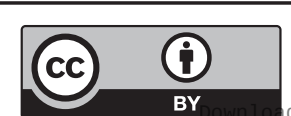

This work is licensed under a Creative Commons Attribution 4.0 International License. 
factor 1 (IGF-1) levels in only 40-50\% of patients with macroadenomas, with higher rates of $>85 \%$ in the less frequent microadenomas (2). Long-acting somatostatin analogs (SSAs) are well-established treatments for acromegaly when disease persists after surgery and are also used as first-line treatment if surgery is refused, contraindicated or unlikely to succeed $(1,3)$.

Although long-acting SSAs can establish hormonal remission and induce tumor shrinkage, around 30\% of patients have a poor response $(1,3)$. Hormonal response to SSAs has been linked to adenoma distribution of cytoplasmic keratin. Sparsely granulated (SG) tumors are more likely than densely granulated (DG) tumors to be SSA-resistant, larger, more invasive and associated with a higher Ki-67 labeling index and lower serum levels of IGF-1 $(4,5,6)$. The increased SSA responsiveness of DG vs SG tumors has been linked to high expression of somatostatin receptor 2A (7). Nevertheless, primary clinical decisions are made without knowledge of histological granulation patterns or receptor distribution.

MRI could provide non-invasive insight into these features and their associations with clinical outcomes. T2 signal intensity of GH-secreting pituitary adenomas varies from hyper-, iso-, to hypo-intense, in relation to surrounding tissue $(8,9)$. DG tumors tend to be T2 signal hypointense (9). Hypointense tumors are also smaller, with less cavernous sinus invasion, less frequent optic chiasm compression and higher IGF-1 levels than iso-/hyperintense tumors (10). T2 signal hypointensity correlated with better IGF-1 response in patients receiving SSAs for persistent disease after surgery or as primary medical treatment $(11,12)$. In another cohort receiving primary SSA treatment, high T2 signal intensity correlated negatively with reductions in GH, IGF-1 and adenoma volume (13). Histology in these studies confirmed that hyperintense tumors were more likely to be SG than DG or of intermediate granular density $(11,13)$. The definition of T2 signal hypointensity of GH-secreting adenomas, however, varies between studies. T2 signal intensity has been assessed by qualitative (visual) or quantitative methods, and adenoma tissue compared against gray or white cerebral matters, as well as normal pituitary tissue $(10,11,12,13)$.

In PRIMARYS, $63 \%$ of 89 patients with previously untreated GH-secreting pituitary macroadenomas had tumor volume reduction (TVR) $\geq 20 \%$ (tumor response) after 1 year of primary treatment with the long-acting SSA lanreotide autogel (depot in the USA; Ipsen Pharma Biotech, France) $120 \mathrm{mg}$, and 34\% achieved both GH $\leq 2.5 \mu \mathrm{g} / \mathrm{L}$ and IGF-1 normalization (hormonal control) (14). This homogenous study population - using a fixed dose in previously treatment-naive patients and thereby avoiding any influence of prior surgery and/or SSA dose changes - offers the opportunity to analyze the association of MRI T2 signal reading and response to treatment. Accordingly, the primary objective of these post hoc analyses of PRIMARYS data was to determine whether the initial MRI T2 signal intensity of GH-secreting pituitary adenomas was predictive of achieving hormonal control at week 48/last visit available (LVA). The association between T2 signal intensity at baseline and achievement of tumor response at week 48/LVA was also investigated. Given that various approaches to its assessment have been reported, T2 signal intensity of GH-secreting adenomas was defined using three different methods.

\section{Subjects and methods}

\section{Study design and interventions}

PRIMARYS was a prospective multicenter, open-label, singlearm phase IIIb study in which patients with GH-secreting pituitary macroadenomas received primary medical treatment with lanreotide autogel at a fixed dose of $120 \mathrm{mg}$ every 4 weeks for 1 year (14). Each pituitary MRI collected during the main PRIMARYS study (as previously described (14)) was re-read by a single neuroradiologist to determine T2 signal intensity and assess complementary information. The PRIMARYS study, from which this post hoc analysis is derived, is registered with EudraCT (EudraCT2007-000155-34) and ClinicalTrials.gov (NCT00690898). As previously described, patients provided written informed consent before study start, the trial was conducted with the Declaration of Helsinki, Good Clinical Practice guidelines and all local regulatory requirements before trial initiation, and the protocol, its amendments, consent form, study questionnaires and the patient information leaflet were approved by institutional review boards (14).

\section{Study population}

Men and women (age, 18-75 years) with acromegaly and pituitary macroadenoma (diameter $\geq 10 \mathrm{~mm}$ ) were eligible for inclusion in PRIMARYS if they were treatment-naïve, had elevated GH and IGF-1 levels and had no visual field defects. Full inclusion and exclusion criteria have been published (14). 


\section{Assessments and objectives}

MRI scans were reviewed by a neuroradiologist blinded to patient identity and response to treatment. Three methods were used to determine the T2 signal intensity of each adenoma relative to surrounding tissue (Fig. 1). The qualitative method comprised visual assessment, with gray matter as the comparator tissue. Of the two quantitative methods, one used the signal intensity ratio of the adenoma and gray matter (signal-ratio method), and the other was a three-tissue assessment (adenoma, gray matter and white matter tissues). Each tumor was rated as hypointense, isointense or hyperintense according to each method, as defined in Fig. 1, and exemplified in Fig. 2. Images were reviewed in two sequential batches: firstly, by visual assessment for $\mathrm{T} 2$ signal intensity and to identify extrasellar extension parameters (EEPs (suprasellar, intrasphenoidal and cavernous sinus extensions), which were not assessed in the main PRIMARYS study) at baseline and secondly, by the two quantitative methods,
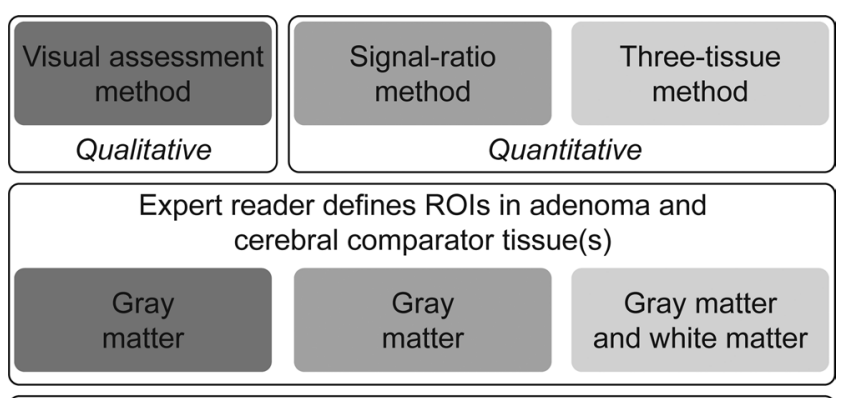

T2 signal intensity compared between adenoma and comparator tissue ROls

Compared
visually

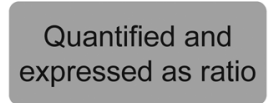

Quantified and Hyperintense adenoma

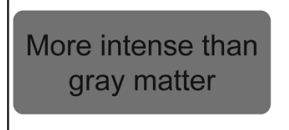

$\mathrm{SR} \geq 1.2$ compared

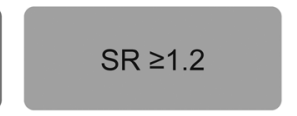

Isointense adenoma
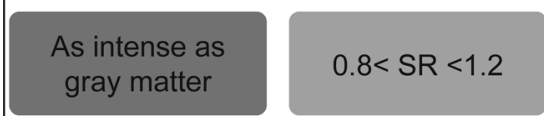

Hypointense adenoma

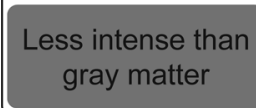

$\mathrm{SR} \leq 0.8$ signal intensity

at baseline, week 24 and week 48/LVA, with the reader additionally blinded to the time point. Of note, no adenomas were purely cystic in our cohort. In case of heterogeneous adenoma with both cystic and solid components, T2 signal intensity was assessed only in the solid component of GH-secreting adenomas. Cystic component, as defined as intratumoral areas presenting with high T2 signal intensity and corresponding nonenhancing low-T1 signal intensity, was excluded from all visual and quantitative analyses.

GH and IGF-1 levels were assayed centrally in the main study and used to identify patients achieving hormonal control (GH $\leq 2.5 \mu \mathrm{g} / \mathrm{L}$ and normalized IGF-1) (14). Achievement of tumor response was defined as TVR $\geq 20 \%$ at week $48 /$ LVA.

The primary objective of these post hoc analyses was to determine whether the T2 signal intensity of GH-secreting pituitary adenomas at baseline was predictive for hormonal control at week 48/LVA after primary medical treatment with lanreotide autogel $120 \mathrm{mg}$ in the
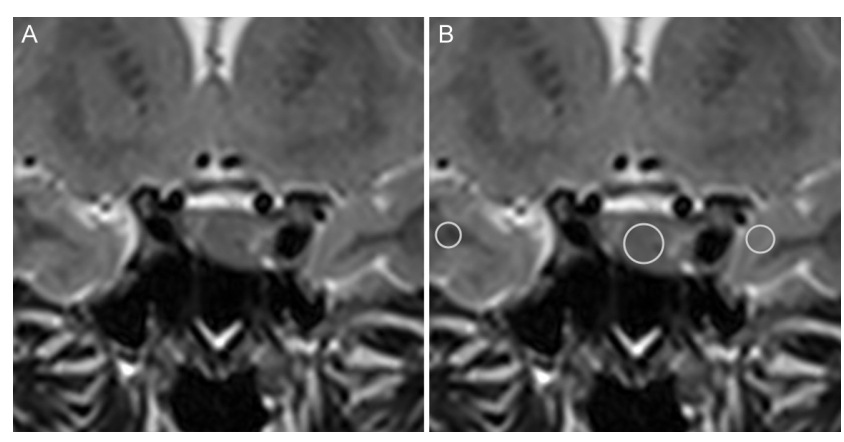

\section{Figure 2}

Classification of $\mathrm{T} 2$ signal intensity of a pituitary macroadenoma by one qualitative and two quantitative methods. (A) Qualitative analysis: visual assessment method; (B) quantitative analysis: signal-ratio and three-tissue methods. Coronal T2-weighted image of a GH-secreting pituitary adenoma at baseline in the PRIMARYS study. (A) By qualitative analysis, the adenoma is hypointense (the signal appears less intense than the gray matter). (B) ROls (circled) are identified and signal intensity is quantified (left to right): white matter (290), adenoma (349), gray matter (451). According to the signal-ratio method, the adenoma is hypointense because the ratio of adenoma/gray matter signals $=0.77$. However, according to the three-tissue method, the T2 signal intensity of the adenoma is isointense because the signal of the adenoma (349) lies between those of white matter (290) and gray matter (451). GH, growth hormone; MRI, magnetic resonance imaging; ROIs, regions of interest.

\section{Figure 1}

Methods used to assess MRI T2-signal intensities of macroadenomas in patients with acromegaly. MRI, magnetic resonance imaging; ROI, region of interest; SR, signal ratio. 
PRIMARYS study. Secondary objectives of these post hoc analyses comprised: description of MRI characteristics of GH-secreting pituitary adenomas (T2 signal intensity, EEPs) at baseline; description of the evolution in T2 signal intensity during the study; analysis of the associations between the presence of EEPs at baseline and achievement of hormonal control at week 48/LVA; analysis of the associations between $\mathrm{T} 2$ signal intensity and EEPs at baseline and achievement of separate hormonal control parameters at week 48/LVA $(\mathrm{GH} \leq 2.5 \mu \mathrm{g} / \mathrm{L}$, normalized IGF-1); analysis of the associations between T2 signal intensity and EEPs at baseline and achievement of tumor response at week 48/LVA and analysis of the extent to which MRI characteristics at baseline are associated with the variation between patients in change from baseline to week 48/LVA in GH and IGF-1 levels and in tumor volume.

\section{Statistical analysis}

Patients from the intention-to-treat (ITT) population (patients receiving at least one injection of study medication and with at least one baseline assessment of tumor volume (14)) were included in the post hoc analyses. Descriptive summary statistics ( $n$, mean, standard deviation, median, first and third quartiles (Q1, Q3), minimum, maximum, or frequency counts and proportions (with Clopper-Pearson 95\% confidence intervals; CI)) were computed. Normality was assessed using the Kolmogorov-Smirnov test, and non-normal data are presented as median (Q1, Q3) rather than mean (s.D.). Statistical tests were performed using a two-sided $\alpha$-level of 0.05 without any multiplicity correction. Accordingly, all statistical tests were exploratory in nature and hypothesisgenerating. Statistical analysis was performed using SAS version 9.2.

Stepwise multivariate analyses were used to investigate factors predictive for hormonal control at week 48/LVA, focusing on T2 signal intensity at baseline. Univariate logistic regression analyses were first conducted to identify suggestive associations $(P<0.2)$. Multivariate logistic regression models were then used, in which T2 signal intensities were retained and to which 'significant' factors from the univariate analyses were added (no interaction was tested). Factors investigated comprised baseline variables: T2 signal intensity, GH level, IGF-1 level, tumor volume and EEPs. $P$ values (chi-square) were generated using likelihood ratio tests, and odds ratios were estimated, with Wald 95\% CIs.

Similar logistic regression was used to investigate factors predictive for tumor response at week 48/LVA.
Linear regressions were also performed to assess whether T2 signal intensity at baseline is associated with variation in change from baseline to week 48/LVA in levels of $\mathrm{GH}$ or of IGF-1, and with variation in tumor volume. The Bhapkar test was used to compare distributions between baseline and week 48 or LVA in signal intensity category.

\section{Results}

\section{Patient disposition and baseline characteristics}

Of 90 patients enrolled in PRIMARYS, one was excluded from the ITT population because he lacked a centralized baseline tumor volume assessment (14). All patients in the ITT population had baseline MRI available for assessment of EEPs. The baseline T2 signal intensity data were available for 85 patients for each method and are included in this analysis, of whom 30 achieved hormonal control ( $\mathrm{GH} \leq 2.5 \mu \mathrm{g} / \mathrm{L}$ and normalized IGF-1) and 53 achieved a tumor response (TVR $\geq 20 \%$ ) at week $48 / \mathrm{LVA}$. Baseline characteristics according to T2 signal intensity category by visual assessment are shown in Table 1; data for the quantitative methods are shown in Supplementary Table 1 (see section on supplementary data given at the end of this article). Baseline characteristics according to achievement of hormonal control by study end were reported previously (15).

There were no notable differences in baseline characteristics among the hypointense, isointense and hyperintense tumor groups (irrespective of assessment method). The exception was that baseline tumor volume tended to be lower in the hypointense tumor group than the other groups, a difference that was most pronounced when tumors were categorized by visual assessment (median (95\% CI) tumor volume: hypointense, 1158 (959-1810) $\mathrm{mm}^{3}$; isointense, 2017 (1388-4066) $\mathrm{mm}^{3}$; hyperintense, 4767 (1872-22 725) $\mathrm{mm}^{3}$; Fig. 3).

\section{MRI findings at baseline}

The three methods resulted in different proportions of patients assigned to each T2 signal intensity category. Overall, over half the adenomas (59\%) were classified as hypointense at baseline with the visual assessment method, considerably more than with either of the quantitative methods (signal-ratio method, 36\%; three-tissue method, 20\%). Approximately half the adenomas were classified as isointense by the quantitative methods, compared with $36 \%$ by visual assessment. More adenomas (33\%) were classified as hyperintense with the three-tissue method 
Table 1 Demographic and disease characteristics at baseline according to visual T2-weighted signal intensity. * Tumor volume is presented in Fig. 3. Data are expressed as mean (S.D.), unless stated otherwise, from the ITT population with available baseline T2 signal intensity data. Median (Q1, Q3) are presented for non-normal data. Percentages are based on the number of patients with available data.

\begin{tabular}{l}
\hline Characteristics \\
\hline Age (years) \\
Men:women $(n(\%))$ \\
BMI $\left(\mathrm{kg} / \mathrm{m}^{2}\right)$ \\
$\mathrm{GH}$ level $(\mu \mathrm{g} / \mathrm{L})^{\dagger}$ \\
IGF-1 level $(\mu \mathrm{g} / \mathrm{L})$ \\
No. of days since acromegaly \\
diagnosis \\
Median $(\mathrm{Q} 1-\mathrm{Q} 3)$
\end{tabular}

\begin{tabular}{c} 
All $(n=85)$ \\
\hline $50.0(12.3)$ \\
$39(45.9): 46(54.1)$ \\
$27.5(4.5)$ \\
$8.3(3.7-15.2)$ \\
$793.5(291.4)$ \\
68 \\
$99(61-133)$
\end{tabular}

\begin{tabular}{l}
\hline Hypointense $(n=50)$ \\
\hline $51.4(12.2)$ \\
$25(50.0): 25(50.0)$ \\
$27.6(4.5)$ \\
$9.7(4.3-16.9)$ \\
$820.6(293.1)$ \\
35 \\
$103(61-148)$
\end{tabular}

\begin{tabular}{l}
\hline Isointense $(n=31)$ \\
\hline $47.9(12.9)$ \\
$11(35.5): 20(64.5)$ \\
$27.7(4.7)$ \\
$8.0(3.5-13.2)$ \\
$764.8(297.1)$ \\
30 \\
$98(70-119)$ \\
\hline
\end{tabular}

\begin{tabular}{c} 
Hyperintense $(n=4)$ \\
\hline $48.0(7.2)$ \\
$3(75.0): 1(25.0)$ \\
$25.4(2.8)$ \\
$5.4(2.2-7.6)$ \\
$677.3(230.5)$ \\
3
\end{tabular}

*As assessed visually in comparison to gray matter; baseline data according to categorization by the signal-ratio and three-tissue methods are shown in Supplementary Table 1; ${ }^{\dagger}$ Data preseted as meadian (Q1-Q3); ${ }^{\ddagger}$ relative to baseline.

BMI, body mass index; GH, growth hormone; IGF-1, insulin-like growth factor 1; ITT, intention-to-treat; LVA, last visit available; Q, quartile; s.D., standard deviation.

than with the other two methods (visual assessment, 5\%; signal-ratio method, 12\%). An example of an MRI scan classified differently with different assessment methods is shown in Fig. 2.

Data on EEPs at baseline by status at week 48/LVA were available for 88 patients for hormonal control (hormonal

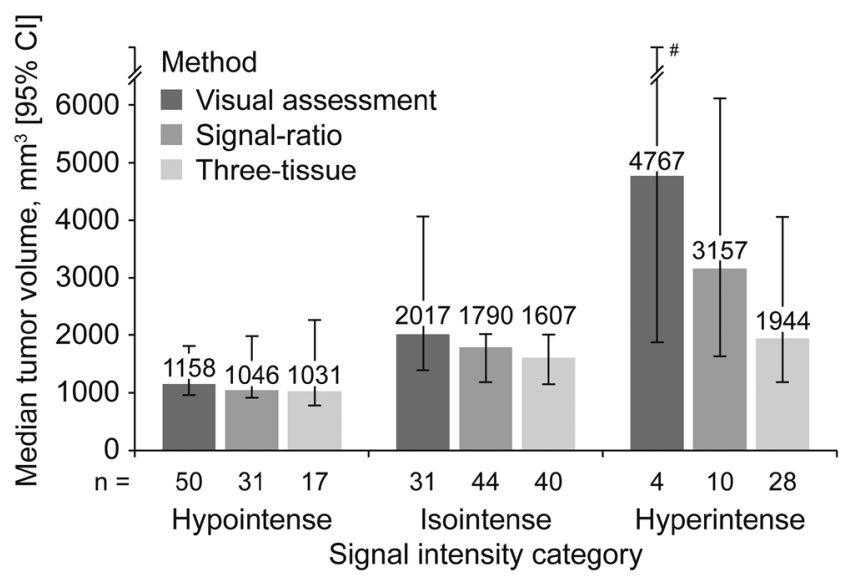

\section{Figure 3}

Tumor volume of $\mathrm{GH}$-secreting pituitary macroadenomas at baseline in each MRI T2 signal intensity category as defined by three methods. For the qualitative visual assessment method and the quantitative signal-ratio method, gray matter was used as a comparator for adenoma tissue T2 signal intensity. The quantitative three-tissue method used gray and white matter as comparator tissues for adenoma tissue T2 signal intensity. Tumor volumes are from the measurements made for analysis of baseline images at the end of the PRIMARYS study (not the initial measurements made to assess fulfillment of inclusion criteria). ${ }^{\#}$ Upper $95 \% \mathrm{Cl}$ limit $=17$ 958. Cl, confidence interval; MRI, magnetic resonance imaging. control, $n=30$; non-controlled, $n=58$ ) and 89 patients for tumor response (tumor response, $n=56$; non-response, $n=33)$. Of the 88 patients with complete data, baseline MRI of the adenoma showed suprasellar extension for 23 (26\%) patients, intrasphenoidal extension for 27 (31\%) patients and cavernous extension for 28 (32\%) patients. Extrasellar extension tended to be more frequent in non-controlled vs controlled patients (e.g. suprasellar: 31\% vs 17\%), although 95\% CIs were overlapping (Supplementary Fig. 1).

\section{Association between MRI findings and response to treatment}

Qualitative assessment

Using the visual assessment method, of the patients diagnosed with a hypointense adenoma at baseline, a large proportion of them demonstrated hormonal control (40\%) and a tumor response (76\%) to treatment by week 48/LVA (Fig. 4A and B). Of the patients diagnosed with an isointense adenoma at baseline using the visual assessment method, 27\% demonstrated hormonal control and $42 \%$ demonstrated tumor response (Fig. 4A and B). Of the patients diagnosed visually with a hyperintense adenoma at baseline, 50\% demonstrated hormonal control and tumor response (Fig. 4A and B). However, it should be noted that this patient cohort consisted of four patients.

\section{Quantitative assessments}

Using the quantitative methods, similar hormonal and tumor response values were observed in hypointense adenomas compared with the visual assessment method (Fig. 4C, D, E and F). The proportion of patients with 

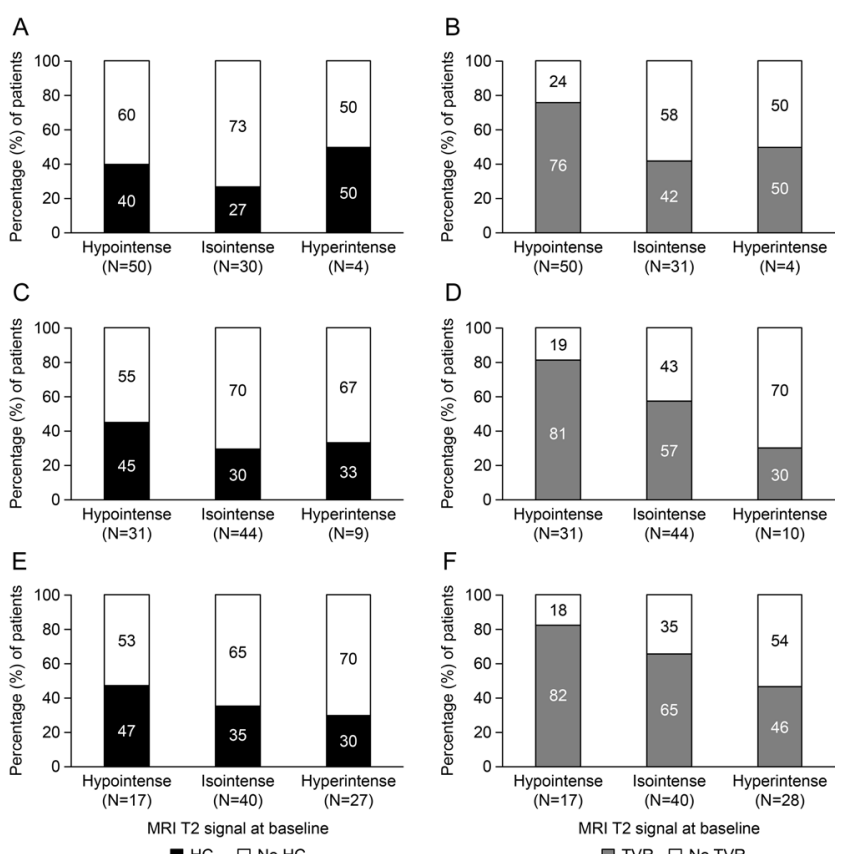

\section{Figure 4}

Proportion of patients that gained hormonal control (A, C and E) and tumor volume response (B, D and F) according to MRI T2 signal at baseline. Data are shown based upon three methods: visual assessment ( $A$ and $B$ ), signal-ratio (C and $D$ ) and the three-tissue method ( $\mathrm{E}$ and $\mathrm{F}$ ). Hormonal control is defined as $\mathrm{GH} \leq 2.5 \mu \mathrm{g} / \mathrm{L}$ and IGF-1 levels within normal range. Tumor response is defined as TVR $\geq 20 \%$ from baseline. The visual assessment and signal-ratio methods used gray matter as a comparator for adenoma tissue; the three-tissue method used gray and white matter as comparator tissues. One patient without hormonal status due to missing post-baseline IGF-1 levels had an isointense adenoma according to the qualitative method and a hyperintense adenoma using the quantitative methods (signal-ratio and three-tissue). IGF-1, insulin-like growth factor 1; LVA, last visit available; TVR, tumor volume reduction.

an isointense adenoma at baseline who demonstrated hormonal control and tumor response using the quantitative methods tended to be higher for both the signal ratio and three-tissue method compared with the visual assessment method (Fig. 4C, D, E and F). Between 30 and 33\% of patients with hyperintense adenomas gained hormonal control following treatment as assessed by the quantitative methods (Fig. 4C and E). The proportion of patients with hyperintense tumors who had a tumor response was slightly higher in the threetissue method (46\%) than the signal-ratio method (30\%) (Fig. 4D and F).

\section{Factors predictive for treatment response}

In univariate analyses, T2 signal intensity at baseline was not significantly associated with hormonal control at week 48/LVA (for all methods of assigning intensity category, data not shown) (primary endpoint). The presence of any EEPs at baseline was also not associated with hormonal control.

Univariate associations of T2 signal intensity were also investigated with GH levels and IGF-1 levels as separate endpoints. T2 signal intensity at baseline was significantly associated with $\mathrm{GH} \leq 2.5 \mu \mathrm{g} / \mathrm{L}$ at week $48 / \mathrm{LVA}$, as defined by visual assessment $(P<0.001$; OR (hypo- vs iso-intense $)=7.27 ; 95 \% \mathrm{CI}: 2.57-20.56)$ and signal-ratio methods $(P=0.004$; OR (hypo- vs iso-intense $)=4.67 ; 95 \%$ CI: 1.39-15.66), with no significant association using the three-tissue method. Among EEPs, presence of cavernous extension was the only one significantly (negatively) associated with $\mathrm{GH} \leq 2.5 \mu \mathrm{g} / \mathrm{L}$ at week $48 / \mathrm{LVA}(P=0.021$; $\mathrm{OR}=0.34$; 95\% CI: 0.13-0.86).

For IGF-1 normalization at week 48/LVA, no significant univariate association was found with T2 signal intensity at baseline by visual assessment, whereas borderline significant associations were identified with T2 signal intensity at baseline as defined by signal-ratio $(P=0.082$; OR (hypo- vs iso-intense $)=2.90$; 95\% CI: 1.11-7.55) and three-tissue methods $(P=0.053$; OR (hypo- vs iso-intense) $=3.40 ; 95 \% \mathrm{CI}$ : 1.04-11.17). No significant associations with IGF-1 normalization were identified for EEPs. In contrast to hormonal control, T2 signal intensity at baseline was significantly associated with tumor response (TVR of $\geq 20 \%$ ) at week 48/LVA in univariate analyses, regardless of the method used (visual assessment, $P=0.008$; OR (hypervs iso-intense) $=1.38$; 95\% CI: 0.17-11.15; OR (hypovs iso-intense) $=4.38$; 95\% CI:1.67-11.50-; signal-ratio method, $P=0.008$; OR (hyper- vs iso-intense) $=0.33 ; 95 \%$ CI: 0.07-1.43; OR (hypo- vs iso-intense) $=3.17$; 95\% CI: 1.08;9.25-; three-tissue method, $P=0.043$; OR (hypervs iso-intense) $=0.47 ; 95 \% \mathrm{CI}$ : $0.17 ; 1.25-$; OR (hypo- vs iso-intense) $=2.51 ; 95 \%$ CI: 0.62-10.25). Presence of any EEPs at baseline was not significantly associated with tumor response. $\mathrm{GH}$ at baseline reached the threshold for significance $(P<0.2)$ on univariate analysis and was therefore included with T2 signal in the multivariate models for tumor response $(P=0.073$, OR $=1.03$; 95\% CI: 0.99-1.06).

In multivariate analyses, the odds of achieving a tumor response at week 48/LVA were six times higher for hypovs iso-intense as defined by visual assessment $(\mathrm{OR}=6.15$; 
95\% CI: $1.36-27.88) ; P=0.019)$ or the signal-ratio method (OR=6.43; 95\% CI: $1.31-31.58 ; P=0.022$ ), but were not significantly different using the three-tissue assessment method $(\mathrm{OR}=2.40 ; \quad 95 \%$ CI: $0.36-15.91 ; \quad P=0.36)$. Differences for hyper- vs iso intensity (by any method) did not reach significance in multivariate analysis.

\section{Associations between $\mathrm{T} 2$ signal intensity and change from baseline in GH, IGF-1 and TVR}

In univariate analyses, $\mathrm{T} 2$ signal intensity by visual assessment or three-tissue methods was not significantly associated with the change from baseline to week 48/LVA in GH level, although by the signal-ratio method the association was considered suggestive (according to the $P<0.20$ threshold; $P=0.16$ ).

For change from baseline to week 48/LVA in IGF-1 level, univariate analyses found significant associations with T2 signal intensity by visual assessment $(P=0.005)$, signal-ratio $(P=0.127)$ and three-tissue methods $(P=0.024)$. In the multivariate (visual assessment) model, there was an estimated $65 \mu \mathrm{g} / \mathrm{L}$ greater reduction in IGF-1 level between baseline and week 48/LVA for patients with hypo- vs isointense tumors $(P=0.003)$ (Supplementary Table 2 (data from the other methods not shown)).

In univariate analyses of TVR from baseline to week 48, significant associations $(P<0.0001)$ were identified with T2 signal intensity (by each method). In the visual assessment model, there was an estimated additional 16.8\% (SE 4.55; $P=0.0004$ ) TVR by week 48/LVA for patients with hypo- vs iso-intense tumors.

\section{Change in T2 signal intensity during the study}

In order to assess whether SSA treatment results in any change in MRI T2 signal intensity, and to further understand the nature of T2 signal intensity, assessments were made post baseline. Most adenomas were assigned to the same signal intensity category at baseline, week 24 and week 48 , whether assessed by the signal-ratio (hypointense: 25\% (week 24) and 29\% (week 48); isointense: 33\% (week 24) and 35\% (week 48); hyperintense: $4 \%$ (week 24) and 4\% (week 48)) or three-tissue (hypointense: $10 \%$ (week 24) and 10\% (week 48); isointense: 32\% (week 24) and $37 \%$ (week 48 ); hyperintense: $22 \%$ (week 24 ) and $21 \%$ (week 48)) methods (Fig. 5). Nonetheless, 2 (3\%) adenomas shifted from hypo- to hyperintense categories from baseline to week 48 (both methods), 10\% shifted from hypo- to iso-intense (both methods) and $4 \%$ (signal-ratio) or $7 \%$ (three-tissue) shifted from iso- to hyperintense categories. However, comparisons between baseline and week 48 or LVA in signal intensity category did not reach statistical significance (for either method).

\section{Discussion}

Our post hoc analysis of MRI data from the PRIMARYS study suggests that assessment of $\mathrm{T} 2$ signal intensity of untreated GH-secreting pituitary macroadenomas at baseline can contribute to predicting the effect of primary treatment with lanreotide autogel. In brief, although signal intensity was not significantly associated with achievement of hormonal control (defined as $\mathrm{GH} \leq 2.5 \mu \mathrm{g} / \mathrm{L}$ and normalized IGF-1, in accordance with PRIMARYS),

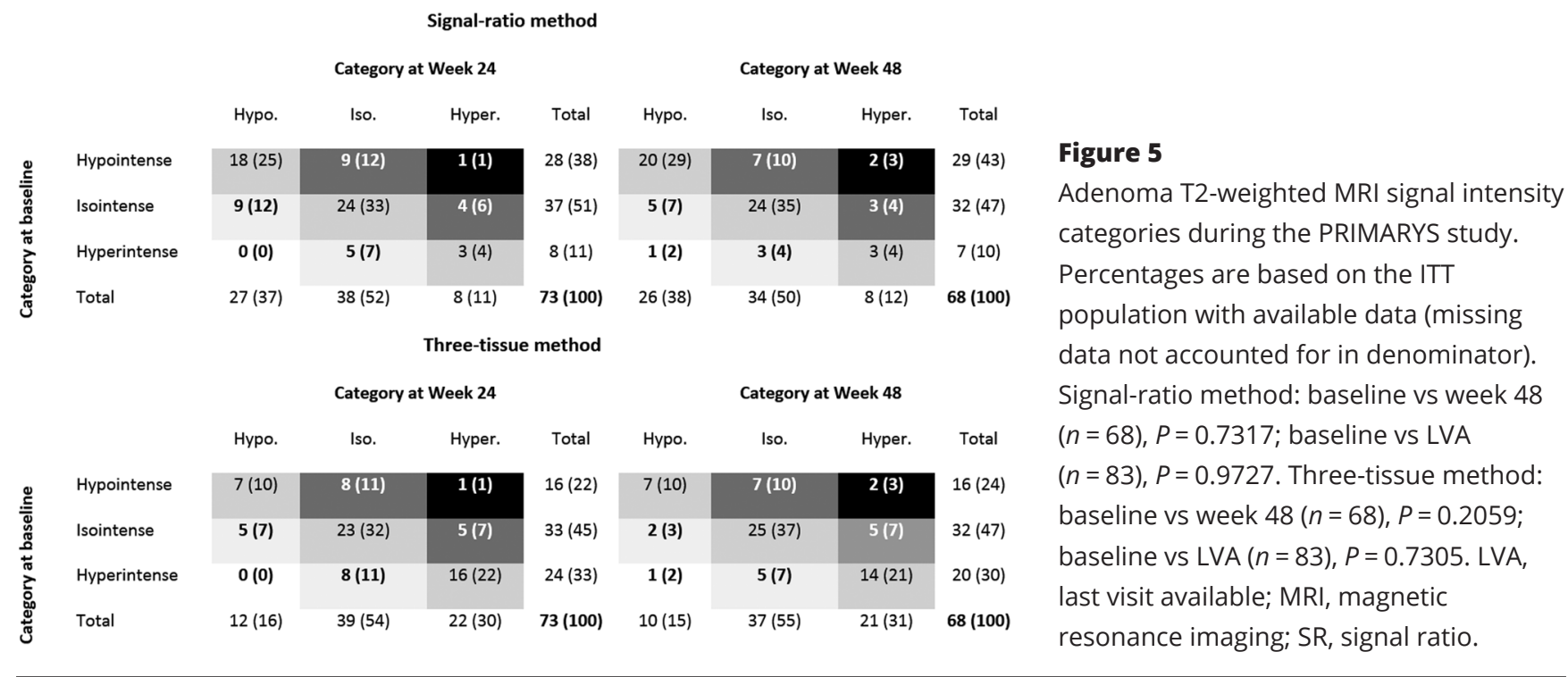


significant associations with other responses to treatment were identified, most notably with tumor response.

Tumor response (TVR $\geq 20 \%$, which was achieved by $62 \%$ of the population at week $48 / \mathrm{LVA}$ ) was estimated in multivariate analysis to be six times more likely for hypointense vs isointense tumors (visual assessment, $\mathrm{OR}=6.15,95 \%$ CI $(1.36 ; 27.88))$. T2 signal intensity at baseline also showed significant univariate associations with the extent of TVR from baseline. Hypointense tumors underwent an estimated additional 17\% TVR from baseline to study end, compared with isointense tumors (visual assessment).

We noted substantial differences in the frequency of each MRI T2 signal intensity category as defined by the three methods used. By visual assessment in comparison with gray matter, 59\% of patients in the PRIMARYS study had hypointense tumors. This proportion is similar to the $53 \%$ reported in another study that also used visual assessment in recently diagnosed patients with acromegaly (10). In that study, adenomas (including microadenomas) were compared against normal pituitary tissue or gray matter when normal pituitary was not visible (10). Normal pituitary would have been a less practical comparator tissue in our study (including exclusively macroadenomas), as it is often not visible in MRI. In contrast to the proportion by visual assessment, the signal-ratio and three-tissue methods identified 36 and $20 \%$ of tumors in our study as hypointense, which compares with the $27 \%$ reported by Heck et al. (4) and suggests that methodological differences between studies account for a large part of the different reported frequencies of hypointense tumors.

Our study did not find any notable differences in baseline characteristics between tumors classified as hypo-, iso- and hyperintense by any of the methods. A trend toward smaller tumors in the hypointense group agrees with previous reports (10) but did not reach significance.

The primary analysis did not identify significant associations between $\mathrm{T} 2$ signal intensity and the strict binary outcome of hormonal control that was a preplanned endpoint of PRIMARYS ( $\mathrm{GH} \leq 2.5 \mu \mathrm{g} / \mathrm{L}$ and IGF-1 within normal range), which was achieved by $35 \%$ of the study population at week 48/LVA. When univariate associations of T2 signal intensity with GH control or IGF-1 control were analyzed separately, hypo- vs isointensity by visual assessment at baseline was associated with achievement of GH $\leq 2.5 \mu \mathrm{g} / \mathrm{L}$ at week $48 / \mathrm{LVA}$, but not with IGF-1 normalization. In univariate analysis of change from baseline, however, hypo- vs isointensity by visual assessment at baseline was not significantly associated with change in GH, but was significantly associated with change in IGF-1. In multivariate analysis including IGF-1 at baseline, IGF-1 levels were estimated to reduce by an additional $65 \mu \mathrm{g} / \mathrm{L}$ for hypointense vs isointense tumors (visual assessment, $P=0.003$ ). Overall, the proportion of patients who demonstrated a response to SSA treatment in terms of both hormonal control and tumor response seems to be greater in those patients classified as having a hypointense adenoma at baseline, regardless of the method used. These data support previous studies that suggested that hypointense tumors are more likely to respond to SSAs, in which reductions in $\operatorname{IGF}-1(4,12)$ or in GH and IGF-1 as two continuous outcomes (13) were examined. To our knowledge, the exact mechanism underlying the relationship of T2 hypointensity and tumor response to SSA therapy is not completely understood. The histopathologic predictors of SSA responsiveness of DG (hypointense) tumors may include an increased expression of somatostatin receptor $2 \mathrm{~A}\left(\mathrm{SSTR} 2_{\mathrm{A}}\right.$ ) subtype and high adenylate cyclase levels associated with G stimulatory protein mutation. Conversely, the lower SSA responsiveness of SG (hyperintense) tumors has been associated with lower SSTR2 ${ }_{\mathrm{A}}$ expression, somatic mutation of the GH receptor and decreased E-cadherin immunoreactivity (7).

These findings are the result of post hoc analyses and are limited in their power to draw significant conclusions. Although T2 signal intensity was significantly associated with achievement of a tumor response, the 95\% CIs were wide, indicating low confidence in the estimated size of the effect. This analysis could not address the natural course of GH-secreting macroadenoma T2 MRI intensity. The addition of a placebo arm would have been unethical, however, given the known efficacy of SSA therapy for acromegaly. It is also unlikely that the natural course of GH-secreting macroadenoma T2 MRI intensity could have been determined over a 48-week study. The PRIMARYS cohort excluded patients with the less frequent microadenomas, which may be considered a limitation of our study. Conversely, many features of the primary study comprise strengths of our analysis. The homogenous and sizable study population, treated with a fixed dose of lanreotide autogel and with robust data for hormonal levels and tumor volume facilitated the investigation of T2 signal intensities in relation to treatment response. The careful assessment of TVR in particular contrasts with previous studies on this topic. Prior treatments for acromegaly, and SSA dose changes, are potential confounders in predictive analyses, and both were absent in this study. For the visual assessment used in this analysis, the MRI scans were interpreted by only one reader, but the 
findings are consistent with published data. Indeed, to the best of our knowledge, there is no reported assessment of inter-observer differences in the visual assessment of T2 signal intensity of $\mathrm{GH}$-secreting adenomas. In the initial work by Heck and colleagues (11), two radiologists assessed T2 signal intensity of GH-secreting adenomas. However, the final decision was reached by consensus and in their article the kappa statistic (inter-observer agreement) was not evaluated. Additionally, in data from the largest cohort $(n=297)$ describing the MRI appearances of GH-secreting adenomas published to date, T2-signal intensity was visually assessed by a single observer (10). Similarly, a single neuroradiologist performed the MRI analysis in this post hoc analysis of PRIMARYS study data. Therefore, unfortunately we cannot comment on interobserver variability. Furthermore, the visual assessment method does not require quantitative analysis and uses only one tissue for reference, which makes it accessible for routine clinical use and easily reproducible in future studies.

In conclusion, we did not identify any clear advantage of using quantitative vs qualitative assessment of MRI T2 signal intensity. However, we believe the use of three methods to analyze T2 signal intensity in pituitary adenomas in this study is novel and has further enhanced our understanding of MRI assessment in acromegaly. Given the practicality and accessibility of visual assessment of adenoma vs gray matter, we therefore suggest that this method should be preferred for categorizing tumors. Obtaining this information at baseline may help to predict outcomes of lanreotide autogel treatment. Although T2 signal intensity at baseline did not predict the overall achievement of hormonal control, there were significant associations with individual hormonal control parameters. Furthermore, the odds of a tumor response were higher for patients with hypointense compared with isointense GH-secreting macroadenomas.

\section{Supplementary data}

This is linked to the online version of the paper at https://doi.org/10.1530/ EJE-18-0254.

\section{Declaration of interest}

$\mathrm{S} P$ is a member of advisory boards and speaker in workshops for Ipsen and Novartis. J B consults for Ipsen and has previously received lecture fees from Ipsen and Novartis. A H and C S are employed by Ipsen. P C consults and has acted as a speaker for Ipsen, Novartis and Pfizer and is an advisory board member for Ipsen. He is also an editorial board member of the European Journal of Endocrinology. F B and L-D R have nothing to declare.

\section{Funding}

This analysis was supported by Ipsen.

\section{Acknowledgments}

The authors thank the investigators and patients participating in this study, including the PRIMARYS Study Group.

PRIMARYS Study Group: Belgium: L Van Gaal; Czech Republic: J Marek; Finland: P Nuutila, M Välimäki; France: C Ajzenberg, F Borson-Chazot, T Brue, P Caron, O Chabre, P Chanson, C Cortet Rudelli, B Delemer, J-M Kuhn, A Tabarin; Germany: K Badenhoop, C Berg, S Petersenn, C Schöfl, J Schopohl; Italy: S Cannavò, A Colao, L De Marinis; The Netherlands: A Stades, A J van der Lely; Turkey: P Kadıoğlu; UK: J S Bevan, D Flanagan, P Trainer.

Medical writing and submission support was provided by Emma Leah and Richard McDonald of Watermeadow Medical, an Ashfield company, part of UDG Healthcare plc, funded by Ipsen (Emma Leah is now an employee of Ipsen).

\section{References}

1 Katznelson L, Laws ER Jr, Melmed S, Molitch ME, Murad MH, Utz A, Wass JA \& Endocrine Society. Acromegaly: an endocrine society clinical practice guideline. Journal of Clinical Endocrinology and Metabolism 201499 3933-3951. (https://doi.org/10.1210/jc.20142700)

2 Schofl C, Franz H, Grussendorf M, Honegger J, Jaursch-Hancke C, Mayr B, Schopohl J \& participants of the German Acromegaly Register. Long-term outcome in patients with acromegaly: analysis of 1344 patients from the German Acromegaly Register. European Journal of Endocrinology 2013168 39-47. (https://doi.org/10.1530/ EJE-12-0602)

3 Giustina A, Chanson P, Kleinberg D, Bronstein MD, Clemmons DR, Klibanski A, van der Lely AJ, Strasburger CJ, Lamberts SW, Ho KK et al. Expert consensus document: a consensus on the medical treatment of acromegaly. Nature Reviews Endocrinology 201410 243-248. (https://doi.org/10.1038/nrendo.2014.21)

4 Fougner SL, Casar-Borota O, Heck A, Berg JP \& Bollerslev J. Adenoma granulation pattern correlates with clinical variables and effect of somatostatin analogue treatment in a large series of patients with acromegaly. Clinical Endocrinology 201276 96-102. (https://doi. org/10.1111/j.1365-2265.2011.04163.x)

5 Kasuki L, Wildemberg LE, Neto LV, Marcondes J, Takiya CM \& Gadelha MR. Ki-67 is a predictor of acromegaly control with octreotide LAR independent of SSTR2 status and relates to cytokeratin pattern. European Journal of Endocrinology 2013169 217-223. (https://doi.org/10.1530/EJE-13-0349)

6 Larkin S, Reddy R, Karavitaki N, Cudlip S, Wass J \& Ansorge O. Granulation pattern, but not GSP or GHR mutation, is associated with clinical characteristics in somatostatin-naive patients with somatotroph adenomas. European Journal of Endocrinology 2013168 491-499. (https://doi.org/10.1530/EJE-12-0864)

7 Cuevas-Ramos D \& Fleseriu M. Somatostatin receptor ligands and resistance to treatment in pituitary adenomas. Journal of Molecular Endocrinology 201452 R223-R240. (https://doi.org/10.1530/JME-140011)

8 Bonneville JF, Bonneville F \& Cattin F. Magnetic resonance imaging of pituitary adenomas. European Radiology 200515 543-548. (https:// doi.org/10.1007/s00330-004-2531-x)

9 Hagiwara A, Inoue Y, Wakasa K, Haba T, Tashiro T \& Miyamoto T. Comparison of growth hormone-producing and non-growth hormone-producing pituitary adenomas: imaging characteristics and pathologic correlation. Radiology 2003228 533-538. (https://doi. org/10.1148/radiol.2282020695) 
10 Potorac I, Petrossians P, Daly AF, Schillo F, Ben Slama C, Nagi S, Sahnoun M, Brue T, Girard N, Chanson P et al. Pituitary MRI characteristics in 297 acromegaly patients based on T2-weighted sequences. Endocrine-Related Cancer 201522 169-177. (https://doi. org/10.1530/ERC-14-0305)

11 Heck A, Ringstad G, Fougner SL, Casar-Borota O, Nome T, RammPettersen J \& Bollerslev J. Intensity of pituitary adenoma on T2-weighted magnetic resonance imaging predicts the response to octreotide treatment in newly diagnosed acromegaly. Clinical Endocrinology 201277 72-78. (https://doi.org/10.1111/j.13652265.2011.04286.x)

12 Puig-Domingo M, Resmini E, Gomez-Anson B, Nicolau J, Mora M, Palomera E, Martí C, Halperin I \& Webb SM. Magnetic resonance imaging as a predictor of response to somatostatin analogs in acromegaly after surgical failure. Journal of Clinical Endocrinology and Metabolism 201095 4973-4978. (https://doi.org/10.1210/jc.2010-0573)

\section{signal intensity}

13 Heck A, Emblem KE, Casar-Borota O, Bollerslev J \& Ringstad G. Quantitative analyses of T2-weighted MRI as a potential marker for response to somatostatin analogs in newly diagnosed acromegaly. Endocrine 201652 333-343. (https://doi.org/10.1007/s12020-0150766-8)

14 Caron PJ, Bevan JS, Petersenn S, Flanagan D, Tabarin A, Prévost G, Maisonobe P, Clermont A \& PRIMARYS Investigators. Tumor shrinkage with lanreotide Autogel $120 \mathrm{mg}$ as primary therapy in acromegaly: results of a prospective multicenter clinical trial. Journal of Clinical Endocrinology and Metabolism 201499 1282-1290. (https:// doi.org/10.1210/jc.2013-3318)

15 Caron PJ, Petersenn S, Houchard A, Sert C, Bevan JS \& PRIMARYS Study Group. Glucose and lipid levels with lanreotide autogel 120 $\mathrm{mg}$ in treatment-naive patients with acromegaly: data from the PRIMARYS study. Clinical Endocrinology 201786 541-551. (https:// doi.org/10.1111/cen.13285)

Received 29 March 2018

Revised version received 23 November 2018

Accepted 7 December 2018 